\title{
Relationship between cigarette smoking and one leg with eyes closed balance in Japanese men
}

\author{
Takeshi Saito $^{1,2} \cdot$ Nobuyuki Miyatake $^{2} \cdot$ Kenji Nishii $^{3}$
}

Received: 16 February 2015/Accepted: 18 March 2015/Published online: 28 March 2015

(C) The Japanese Society for Hygiene 2015

\begin{abstract}
Objective To investigate the link between cigarette smoking and one leg with eyes closed balance in Japanese men.

Subjects and methods We used data from 4224 Japanese men, aged $43.3 \pm 13.9$ years, in this cross-sectional study. Balance, such as one leg with eyes closed balance was measured. In addition, cigarette smoking habits were obtained by well-trained medical staff. The effect of cigarette smoking on one leg with eye closed balance was evaluated. Results A total of 1613 men (38.2\%) had smoking habit and 1471 men $(34.8 \%)$ regularly exercised. Age-related changes in one leg with eyes closed balance were noted. One leg with eyes closed balance in subjects with cigarette smoking $(30.9 \pm 31.8 \mathrm{~s})$ was significantly lower than those in subjects without cigarette smoking $(32.4 \pm 33.5 \mathrm{~s})$ even after adjusting for confounding factors i.e. age, body weight and exercise habits.

Conclusion Cigarette smoking might be associated with one leg with eyes closed balance in Japanese men.
\end{abstract}

Keywords Cigarette smoking - One leg with eyes closed balance $\cdot$ Exercise habit $\cdot$ Balance $\cdot$ Japanese men

Nobuyuki Miyatake

miyarin@med.kagawa-u.ac.jp

1 Okayama Southern Institute of Health, Okayama Health Foundation, Okayama 700-0952, Japan

2 Department of Hygiene, Faculty of Medicine, Kagawa University, Kagawa 761-0793, Japan

3 Okayama Health Foundation Hospital, Okayama Health Foundation, Okayama 700-0952, Japan

\section{Introduction}

Cigarette smoking is a serious problem for public health, and it has been reported that $34.1 \%$ of men and $9.0 \%$ of women are current smokers in Japan [1]. Cigarette smoking is also a strong risk factor for atherosclerosis and cardiovascular disease in a dose-dependent manner [2].

Levels of maximal oxygen uptake (aerobic exercise level) and muscle strength were recommended as an exercise and physical activity reference quantity for health promotion in 2006 and in 2013 by the Ministry of Health, Labor and Welfare, Japan [3, 4]. We have previously proved that aerobic exercise level is closely associated with cigarette smoking [5], and prohibiting smoking habits was thought to be useful for increasing aerobic exercise level, while smoking habits may affect other physical fitness factors [6, 7]. According to other physical fitness measures i.e. balance, there are few studies that focused on reductions in mortality or the prevention of lifestyle-related diseases, and the optimal level has not been defined.

Cigarette smoking is a critical risk factor for mortality and various diseases, and the ability of balance might be associated with falls and fractures, which are sever problems in an aging society. Therefore, it is important to clarify the relationship between these factors. However, the effect of cigarette smoking on balance in Japanese is not quite clear at present. One leg with eyes closed balance is one of the balance tests which is measured easily for both examiner and examinee, and it does not need special tools with high reliability $[8,9]$.

In this study, we evaluated the effect of cigarette smoking on one leg with eyes closed balance in Japanese men. 


\section{Subjects and methods}

\section{Subjects}

A total of 4224 men $(43.3 \pm 13.9$ years $)$ aged 20-79 years, were enrolled in a cross-sectional study. Subjects met the criteria including evaluation of balance as previously described (Table 1) [6].

Ethical approval for the study was obtained from the Ethical Committee of the Okayama Health Foundation, Okayama, Japan (H25-6-24).

\section{Clinical parameters}

The anthropometric parameters i.e. height, body weight, body mass index (BMI), abdominal circumference and hip circumference, the data on cigarette smoking $[6,7,10]$, exercise habits [11] and one leg with eyes closed balance [12] were also evaluated.

\section{Statistical analysis}

Data are expressed as mean \pm standard deviation (SD) values. A comparison of parameters was evaluated by using the unpaired $t$ test, Chi squared test, one-factor ANOVA, Scheffes'F test. In addition, we used covariance analysis and logistic regression analysis by adjusting confounding factors; where $p<0.05$ was considered to be statistical significance.

\section{Results}

Clinical profiles were summarized in Table 1. A total of 1613 men $(38.2 \%)$ had smoking habits and one leg with eyes closed balance was measured at $31.8 \pm 32.8 \mathrm{~s}$.

We evaluated the changes in one leg with eyes closed balance with aging (Table 2). One leg with eyes closed balance significantly decreased with age. In addition, no subject who achieved $120 \mathrm{~s}$ on one leg with eyes closed balance was observed in the 60's and 70's age range.

We compared clinical parameters including one leg with eyes closed balance between men with and without cigarette smoking (Table 3). Age and exercise habits were significantly lower, and height and body weight were significantly higher in current smokers when compared to non-smokers. No significant difference in one leg with eyes closed balance was noted between subjects with and without cigarette smoking. However, there were significant differences in some confounding factors, and one leg with eyes closed balance in current smokers was significantly lower than that in non-smokers, even after adjusting for confounding factors i.e. age, body weight and exercise habits $(p<0.0001)$.

\section{Discussion}

The main finding of this study was that cigarette smoking was associated with one leg with eyes closed balance in Japanese men.

Michikawa et al. reported that the relation of one leg standing test time with falls and osteoporosis suggests that a decreased one leg standing test time is associated with an increased prevalence of fracture [12]. However, the relationship between cigarette smoking and the balance test was not clearly proved. Ricci et al. investigated the influence of a history of smoking on the physical capacity of elderly people. Among women, ex-smokers were younger and more flexible in comparison with those non-smokers. Among men, ex-smokers were older and walked more slowly than non-smokers. No difference in the balance test outcome was noted in their study [13]. Bishop et al. also showed no difference on the Berg Balance Scale [14] was noted in patients who were attending a gait and balance disorder clinic [15]. In this study, we solely evaluated the relationship between cigarette smoking and balance i.e. one leg with eyes closed balance in Japanese men. By not adjusting for confounding factors, one leg with eyes closed
Table 1 Clinical profiles of enrolled subjects

\begin{tabular}{llll}
\hline & Mean $\pm \mathrm{SD}$ & Minimum & Maximum \\
\hline Number of subjects & 4224 & & \\
Age & $43.3 \pm 13.9$ & 20 & 79 \\
Height $(\mathrm{cm})$ & $169.1 \pm 6.2$ & 145.3 & 190.9 \\
Body weight $(\mathrm{kg})$ & $70.4 \pm 11.7$ & 39.1 & 175.7 \\
Body mass index $\left(\mathrm{kg} / \mathrm{m}^{2}\right)$ & $24.6 \pm 3.6$ & 13.6 & 61.5 \\
Abdominal circumference $(\mathrm{cm})$ & $84.2 \pm 10.2$ & 58.0 & 157.0 \\
Hip circumference $(\mathrm{cm})$ & $94.2 \pm 6.2$ & 71.0 & 145.5 \\
One leg with eyes closed balance $(\mathrm{sec})$ & $31.8 \pm 32.8$ & 1.0 & 120.0 \\
Number of subjects with cigarette smoking & 1613 & & \\
\hline
\end{tabular}


Table 2 Changes in one leg with eyes closed balance with aging

\begin{tabular}{lccl}
\hline & Number of subjects & Mean \pm SD & Number of subjects with 120 second \\
\hline $20-29$ & 854 & $51.0 \pm 39.8$ & 131 \\
$30-39$ & 1026 & $40.4 \pm 34.9$ & $77^{\mathrm{a}}$ \\
$40-49$ & 900 & $29.8 \pm 27.5$ & $23^{\mathrm{ab}}$ \\
$50-59$ & 785 & $20.5 \pm 22.1$ & $10^{\text {abc }}$ \\
$60-69$ & 531 & $11.0 \pm 11.6$ & $0^{\text {abcd }}$ \\
$70-79$ & 128 & $4.9 \pm 3.7$ & $0^{\text {abcd }}$ \\
\hline
\end{tabular}

$p$ values were showed for the post-hoc test after the comparison of the mean values by age groups

a $p<0.05$ vs $20-29$

b $p<0.05$ vs $30-39$

c $p<0.05$ vs $40-49$

d $p<0.05$ vs $50-59$

Table 3 Comparison of clinical parmeters between subjects with and without cigarette smoking

\begin{tabular}{|c|c|c|c|c|c|c|}
\hline & Current smoker mean \pm SD & Non smoker mean \pm SD & $p$ & $p^{*}$ & $p^{* *}$ & $p^{* * *}$ \\
\hline Number of subjects & 1613 & 2611 & & & & \\
\hline Age & $40.7 \pm 12.5$ & $44.8 \pm 14.4$ & $<0.0001$ & & & \\
\hline Height $(\mathrm{cm})$ & $169.6 \pm 5.9$ & $168.7 \pm 6.3$ & $<0.0001$ & & & \\
\hline Body weight (kg) & $70.8 \pm 12.1$ & $70.1 \pm 11.4$ & 0.0485 & & & \\
\hline Body mass index $\left(\mathrm{kg} / \mathrm{m}^{2}\right)$ & $24.6 \pm 3.8$ & $24.6 \pm 3.5$ & 0.9242 & & & \\
\hline Abdominal circumference $(\mathrm{cm})$ & $84.3 \pm 10.4$ & $84.3 \pm 10.1$ & 0.9735 & & & \\
\hline Hip circumference $(\mathrm{cm})$ & $94.2 \pm 6.3$ & $94.2 \pm 6.2$ & 0.7206 & & & \\
\hline One le $g$ with eyes closed balance (sec) & $30.9 \pm 31.8$ & $32.4 \pm 33.5$ & 0.1477 & 0.0030 & $<0.0001$ & $<0.0001$ \\
\hline Number of subjects with 120 seconds & $83(5.1 \%)$ & $158(6.1 \%)$ & 0.2176 & & & \\
\hline Number of subjects with exercise habits & $422(26.2 \%)$ & $1049(40.2 \%)$ & $<0.0001$ & & & \\
\hline
\end{tabular}

Bold values indicate $p<0.05$

$p$ not adjusting

$p^{*}$ adjusting for age

$p^{* *}$ adjusting for age and exercise habits

$p^{* * *}$ adjusting for age, body weight and exercise habits

balance in men with cigarette smoking was not statistically significant different from that in men without cigarette smoking. It is well known that exercise habits are closely associated with muscle strength [16]. Exercise habits were also closely linked to cigarette smoking in this study. Therefore, we have adjusted for confounding factors including exercise habits, and a significant difference of one leg with eyes closed balance was noted between subjects with and without cigarette smoking in this study. We have also reported that the aerobic exercise level defined by the ventilatory threshold was associated with cigarette smoking in Japanese [5]. Taken together, cigarette smoking may affect one leg with eyes closed balance, and giving up smoking might be considered for improving one leg with eyes closed balance in some Japanese men. Wolf B et al. [17] showed that training program improved the Berg Balance Scale in people aged 75 years and older.
Potential limitations still remain in this study. First, our study was a cross sectional and not a longitudinal study. Second, the 4224 men in our study voluntarily underwent measurements: they were therefore more likely to be health-conscious compared with the average person. Third, we could not show a relation between cigarette smoking and one leg with eyes closed balance in women. Fourth, we could not clarify the mechanism of the link between cigarette smoking and one leg with eyes closed balance. Smokers often have lower levels of current and past leisure-time physical activity, hormonal disorders and nutritional deficits [18]. Sedentary physical activity would induce the functional decline of subjects and cigarette smoking accelerates changes in organic functions. In fact, lower aerobic exercise level, muscle strength and flexibility were characteristic features in subjects with cigarette smoking [5-7]. These many possible factors may affect one 
leg with eyes closed balance in this study. However, it seems reasonable to suggest that prohibiting smoking and promoting exercise habits might result in an improvement in one leg with eyes closed balance in some Japanese men. To confirm this finding, further prospective studies are urgently required.

Acknowledgments This research was supported in part by Research Grants from the Ministry of Health, Labor, and Welfare, Japan. We thank Dr. Takeyuki Numata at Okayama Southern Institute of Health, Okayama Health Foundation for his kind contribution to this study.

Conflict of interest There is no conflict of interest.

\section{References}

1. http://www.mhlw.go.jp/file/04-Houdouhappyou-10904750-Kenkou kyoku-Gantaisakukenkouzoushinka/0000032813.pdf (2014.9.18) (in Japanese).

2. Peto R. Smoking and death: the past 40 years and the next 40 . BMJ. 1994;209:937-9.

3. http://www.mhlw.go.jp/shingi/2006/07/dl/s0719-3b.pdf (2014.9.18) (in Japanese).

4. http://www0.nih.go.jp/eiken/info/pdf/sintai2013.pdf (2014.9.18) (in Japanese).

5. Miyatake N, Numata T, Nishii K, Sakano N, Suzue T, Hirao T, et al. Relation between cigarette smoking and ventilatory threshold in the Japanese. Environ Health Prev Med. 2011;16:185-90.

6. Oda K, Miyatake N, Sakano N, Saito T, Katayama A, Nishii K, et al. The effect of cigarette smoking on flexibility in Japanese. Health. 2012;9:570-3.

7. Saito T, Miaytake N, Sakano N, Oda K, Katayama A, Nishii K, et al. Relationship between cigarette smoking and muscle strength in Japanese men. J Prev Med Public Health. 2012;45:381-6.
8. Giorgetti MM, Harris BA, Jette A. Reliability of clinical balance outcome measures in the elderly. Physiother Res Int. 1998;3:274-83.

9. Franchignoni F, Tesio L, Martino MT, Ricupero C. Reliability of four simple, quantitative tests of balance and mobility in healthy elderly females. Aging (Milano). 1998;10:26-31.

10. Miyatake N, Wada J, Nakatsuka A, Sakano N, Teshigawara S, Miyachi M, et al. Serum vaspin levels are associated with physical activity or physical fitness in Japanese: a pilot study. Environ Health Prev Med. 2014;19:200-6.

11. Miyatake N, Miyachi M, Tabata I, Sakano N, Suzue T, Hirao T, et al. Evaluation of ventilatory threshold and its relation to exercise habits among Japanese. Environ Health Prev Med. 2010;15:274-80.

12. Michikawa T, Nishiwaki Y, Takebayashi T, Toyama Y. One-leg standing test for elderly populations. J Orthop Sci. 2009; $14: 675-85$.

13. Ricci NA, Francisco CO, Rebelatto MN, Rebelatto JR. Influence of history of smoking on the physical capacity of older people. Arch Gerontol Geriatr. 2011;52:79-83.

14. Berg K, Wood-Dauphinee S, Williams JI. The balance scale: reliability assessment with elderly residents and patients with an acute stroke. Scand J Rehabil Med. 1995;27:27-36.

15. Bishop ME, Robinson ME, Light KE. Tobacco use and recovery of gait and balance function in older adults. Arch Phys Med Rehabil. 2009;90:1613-8.

16. Miyatake N, Saito T, Miyachi M, Tabata I, Numata T. Evaluation of muscle strength and its relation to exercise habits in Japanese. Acta Med Okayama. 2009;63:151-5.

17. Wolf B, Feys H, Weerdt De, van der Meer J, Noom M, Aufdemkampe G, et al. Effect of a physical therapeutic intervention for balance problems in the elderly: a single-blind, randomized, controlled multicentre trial. Clin Rehabil. 2001;15:624-36.

18. Szulc P, Duboeuf F, Marchand F, Delmas PD. Hormonal and lifestyle determinants of appendicular skeletal muscle mass in men: the MINOS study. Am J Clin Nutr. 2004;80:496-503. 